\title{
MUC13 promotes lung cancer development and progression by activating ERK signaling
}

\author{
YAO PANG ${ }^{1 *}$, YU ZHANG $^{2 *}$, HONG-YI ZHANG ${ }^{1}$, WEN-HAO WANG ${ }^{1}$, \\ GANG JIN $^{1}$, JIA-WEI LIU ${ }^{1}$ and ZI-JIANG ZHU ${ }^{1}$ \\ ${ }^{1}$ Department of Thoracic Surgery No. 2, Gansu Provincial Hospital; ${ }^{2}$ Department of Clinical Medicine, \\ Gansu Health Vocational College, Lanzhou, Gansu 730000, P.R. China
}

Received September 16, 2020; Accepted May 14, 2021

DOI: $10.3892 / \mathrm{ol} .2021 .13155$

\begin{abstract}
Mucin 13 (MUC13) is a glycoprotein that is expressed on the cell surface and participates in the tumorigenesis of multiple malignancies, including pancreatic cancer, colorectal cancer and renal cancer. However, to the best of our knowledge, the expression levels and function of MUC13 in lung cancer progression have not yet been demonstrated. Therefore, the present study examined the expression pattern and regulatory role of MUC13 in lung cancer tumorigenesis. The results demonstrated that MUC13 was highly expressed in lung cancer tissues and cell lines compared with that in normal tissues and cell lines. Functionally, knockdown of MUC13 inhibited cell proliferation and enhanced the apoptosis of A549 and NCI-H1650 lung cancer cells. Furthermore, silencing of MUC13 suppressed the migration and invasion of lung cancer cells. Additionally, a xenograft tumor model demonstrated that knockdown of MUC13 delayed the development of the lung cancer xenograft and suppressed the expression of proliferation marker Ki-67 in tumor tissues. Mechanistically, MUC13 activated the ERK signaling pathway by enhancing the phosphorylation of ERK, JNK and p38 in lung cancer tissues compared with that in normal tissues. Knockdown of MUC13 inhibited the phosphorylation of ERK/JNK/p38 in A549 and NCI-H1650 cells. Overall, these findings suggested that MUC13 could act as an oncogenic glycoprotein to accelerate the progression of lung cancer via abnormal activation of the ERK/JNK/p38 signaling pathway and might serve as a therapeutic target for lung cancer treatment.
\end{abstract}

Correspondence to: Dr Zi-Jiang Zhu, Department of Thoracic Surgery No. 2, Gansu Provincial Hospital, 204 Donggang West Road, Lanzhou, Gansu 730000, P.R. China

E-mail: zhuzijiang2020@163.com

*Contributed equally

Key words: mucin 13, lung cancer, ERK/JNK/p38

\section{Introduction}

Lung cancer is one of the most aggressive malignancies and the leading cause of cancer-associated mortality worldwide $(1,2)$. Lung cancer can be classified into two major histological groups: Small cell lung cancer and non-small cell lung cancer. With the development of medical technologies in the past decades, therapeutic strategies, such as surgery, radiation therapy, chemotherapy and targeted therapy, have been greatly improved along with improvements of the outcome of patients with lung cancer (3). However, the prognosis of patients with lung cancer remains unsatisfactory, especially that in patients with metastasis, recurrence and drug resistance (4-6). Therefore, an improved understanding of lung cancer might be beneficial for the improvement of diagnostic and therapeutic strategies.

Mucin 13 (MUC13) was first identified as a member of the transmembrane glycoprotein mucins and is expressed by epithelial and hematopoietic cells (7). MUC13 could protect against intestinal inflammation, and a deficiency in MUC13 might aggravate the development of severe acute colitis upon treatment with dextran sodium sulfate (8). Increasing evidence has demonstrated that dysregulated MUC13 expression is observed in various tumors, including gastric, ovarian, pancreatic and colon cancer (9-12). In colorectal cancer, MUC13 could activate NF- $\kappa$ B signaling and protect colorectal cancer cells from apoptosis (13). Additionally, MUC13 has been reported to promote the development of intrahepatic cholangiocarcinoma via activation of the EGFR/PI3K/AKT signaling pathway (14). Studies have revealed that MUC13 expression could be post-transcriptionally regulated by microRNAs (miRs), such as miR-145 and miR132-3p $(15,16)$. Serum MUC13 expression has been reported to be elevated in certain carcinomas, such as ovarian cancer and gastric cancer $(9,17)$, and could be developed as a novel biomarker (18). However, to the best of our knowledge, the expression and function of MUC13 in lung cancer remain unknown.

Therefore, to elucidate the function and mechanism of MUC13 in lung cancer, the expression pattern of MUC13 was first investigated in lung cancer tissues and cell lines. Subsequently, MUC13 was silenced in lung cancer cell lines, followed by proliferation, apoptosis, migration and invasion analyses to determine the regulatory role of MUC13 in lung cancer. Furthermore, a xenograft tumor model was applied to confirm the findings at the cell level. Based on 
these investigations, the present study aimed to provide novel insights for the understanding of lung cancer, as well as underlying therapeutic targets for the treatment of lung cancer.

\section{Materials and methods}

Patient specimens. Lung adenocarcinoma cancer tissues and adjacent normal tissues ( $1 \mathrm{~cm}$ from tumor margin) sections were collected from 20 patients (14 male and 6 female patients) aged $46.50 \pm 10.30$ years (range, 28-64 years) who underwent surgical resection at Gansu Provincial Hospital (Lanzhou, China) between May 2018 and April 2019. Patients were enrolled in the present study if they met the following criteria: i) Aged 18 years old; ii) pathology confirmed as lung adenocarcinoma; iii) presented with clear TNM stage (19) and detailed clinical information; and iv) had informed consent to participate this research. Patients were excluded if they met any of the following criteria: i) Complicated with other cancer; ii) congenital lung hypoplasia; iii) had infectious disease; or iv) other lung diseases. The clinical characteristics of the patients are summarized in Table I. The patient specimens were snap-frozen and stored in liquid nitrogen $\left(-196^{\circ} \mathrm{C}\right)$ until reverse transcription-quantitative PCR (RT-qPCR) and western blotting were performed. All patients provided written informed consent. The study was approved by the Institutional Ethical Review Board of Gansu Provincial Hospital (Lanzhou, China; approval no. GSRMYYLL-2019-95).

In silico analysis of MUC13 expression. In the present study, MUC13 expression in lung adenocarcinoma was analyzed using the starBase online tool (version 3.1; http://starbase.sysu.edu. cn/panGeneDiffExp.php) based on 526 cancer samples and 59 normal samples, which were downloaded from The Cancer Genome Atlas project via the Genomic Data Commons Data Portal (http://portal.gdc.cancer.gov/), and the expression values of genes from RNA-seq data were scaled with log2(FPKM+0.01).

Cell culture. A total of four lung cancer cell lines (NCI-H460, squamous carcinoma epithelial cells; H1703, squamous carcinoma epithelial cells; A549, adenocarcinoma epithelial cancer cells; and NCI-H1650, adenocarcinoma epithelial cells) were obtained from The Cell Bank of Type Culture Collection of The Chinese Academy of Sciences. A control cell line [normal lung epithelial cells (NLECs), derived from normal human primary lobar epithelial cells] was purchased from the Shanghai Baiye Biotechnology Center, and human bronchial epithelial (HBE) cells were obtained from Procell Life Science \& Technology Co., Ltd. Cells were cultured in DMEM (HyClone; Cytiva) supplemented with $10 \%$ fetal bovine serum (HyClone; Cytiva) and $1 \%$ penicillin-streptomycin (Thermo Fisher Scientific, Inc.). Cells were maintained in a cell incubator at $37^{\circ} \mathrm{C}$ with $5 \% \mathrm{CO}_{2}$.

Transfection. The MUC13 short hairpin RNAs (shRNAs) and scramble shRNA plasmids cloned into $\mathrm{pLKO} .1$ were purchased from Shanghai GenePharma Co., Ltd. Transfection $(2 \mu \mathrm{g} /$ well of a 6-well plate) was performed in A549 or NCI-H1650 cells using Lipofectamine ${ }^{\circledR} 3000$ (Invitrogen; Thermo Fisher Scientific, Inc.) according to the manufacturer's protocol. Cells were incubated at $37^{\circ} \mathrm{C}$ for $48 \mathrm{~h}$. At $48 \mathrm{~h}$ post-transfection, cells were harvested for the following experiments.
$R T$ - $q P C R$. Total RNA was isolated from tissues samples or cultured NLECs, HBE, NCI-H460, H1703, A549 and NCI-1650 cells using an AccuRef RNA isolation kit (AccuRef Scientific) and reverse-transcribed into cDNA using the QuantiTect Reverse Transcription kit (Qiagen $\mathrm{GmbH}$ ) according to the manufacturers' instructions. qPCR analysis was performed using SYBR master mix (Takara Bio, Inc.) on an ABI 7500 instrument with the following conditions: $94^{\circ} \mathrm{C}$ for $5 \mathrm{~min}$, followed by 40 cycles of $94^{\circ} \mathrm{C}$ for $10 \mathrm{sec}, 58^{\circ} \mathrm{C}$ for $30 \mathrm{sec}$, and $72^{\circ} \mathrm{C}$ for $10 \mathrm{sec}$. The relative gene expression was analyzed using the $2^{-\Delta \Delta \mathrm{Cq}}$ method (20) with $\beta$-actin as the internal control. The primer sequences used were: hsa-MUC13 forward, 5'-CTGCGGATGACTGCCTCAATGG-3' and hsa-MUC13 reverse, 5'-ATTGCTTGTGCTGTGCGTTGC-3'; and hsa- $\beta$-actin forward, 5'-CCTGTGGCATCCACGAA ACT-3', and hsa- $\beta$-actin reverse, 5'-GAAGCATTTGCGGTG GACGAT-3'.

Western blotting. Total protein was extracted from tissues samples or cultured A549 and NCI-1650 cells using RIPA lysis buffer (AccuRef Scientific) and quantified using the BCA method (Thermo Fisher Scientific, Inc.). After boiling with an equal volume of loading buffer, a total of $25 \mu \mathrm{g} /$ lane for each sample was subjected to $12 \%$ SDS-PAGE separation. Protein was transferred to a PVDF membrane (MilliporeSigma) and the membrane was blocked with 5\% non-fat milk (non-phosphorylation-specific) or 5\% BSA (phosphorylation-specific; Sangon Biotech Co., Ltd.) at room temperature for $1 \mathrm{~h}$. Subsequently, the membranes were incubated with primary antibodies overnight at $4{ }^{\circ} \mathrm{C}$, followed by incubation with an HRP-conjugated goat anti-rabbit secondary antibody (dilution, 1:10,000; cat. no. ab6721; Abcam) at room temperature for $1 \mathrm{~h}$. The protein bands were visualized using an enhanced chemiluminescence kit (Pierce; Thermo Fisher Scientific, Inc.). Using $\beta$-actin as the internal control, protein bands were semi-quantified using ImageJ software (version 1.48; National Institutes of Health) and the relative expression of protein was determined and compared with other groups. The primary antibodies used in the present study were: MUC13 rabbit monoclonal antibody (mAb) (dilution, 1:1,000; cat. no. ab235450; Abcam), ERK rabbit mAb (dilution, 1:5,000; cat. no. ab184699; Abcam), phosphorylated (p- ERK (ERK1 phospho T202+ERK2 phospho T185) rabbit mAb (dilution, 1:100; cat. no. ab214036; Abcam), JNK rabbit mAb (dilution, 1:1,000; cat. no. ab179461; Abcam), p-JNK (phospho T183+T183+T221) rabbit mAb (dilution, 1:1,000; cat. no. ab124956; Abcam), p38 rabbit mAb (dilution, 1:2,000; cat. no. ab170099; Abcam) and p-p38 rabbit mAb (dilution, 1:1,000; cat. no. ab178867; Abcam).

Cell Counting Kit-8 (CCK-8) assay. For the CCK-8 assay, A549 cells or NCI-H1650 cells were seeded into 96-well plates (2,000 cells/well). Subsequently, $10 \mu \mathrm{l}$ CCK-8 reagent (Dojindo Molecular Technologies, Inc.) was added to each well at 0, 24, 48 and $72 \mathrm{~h}$ according to the manufacturer's protocol, and the cells were incubated for $2 \mathrm{~h}$ at $37^{\circ} \mathrm{C}$. Next, the absorbance of each well was measured at $450 \mathrm{~nm}$ using an ELX800 microplate reader (BioTek Instruments, Inc.) to evaluate the cell viability.

Colony formation assay. For the colony formation assay, A549 cells or NCI-1650 cells were seeded into 6-well plates at 
Table I. Clinicopathological characteristics of patients $(n=20)$.

\begin{tabular}{lc}
\hline Characteristics & No. of patients \\
\hline Age, years & \\
$<60$ & 7 \\
$\geq 60$ & \\
Sex & 14 \\
Male & 6 \\
Female & \\
TNM stage & \\
T & \\
T1-2 & 8 \\
T3 & 9 \\
T4 & 3 \\
N & \\
N0 & 10 \\
N1 & 2 \\
N2 & 4 \\
N3 & 4 \\
M & \\
M0 & \\
M1a & 18 \\
\hline
\end{tabular}

$\mathrm{T}$, primary tumor; $\mathrm{N}$, regional lymph nodes; $\mathrm{M}$, distant metastasis.

a density of 500 cells/well and cultured for 14 days. Cell colonies were fixed with $100 \%$ methanol at room temperature for 15 min and stained with $1 \%$ crystal violet in $20 \%$ methanol at room temperature for $30 \mathrm{~min}$. Subsequently, the staining solution was removed and cells were washed with slow running water, followed by natural drying and counting under a light microscope (Nicon Eclipase E600; Nikon Corporation).

Apoptosis assay. A549 cells or NCI-1650 cells were washed with ice-cold PBS in triplicate and suspended in $200 \mu \mathrm{l}$ binding buffer at a concentration of $1.0 \times 10^{6}$ cells $/ \mathrm{ml}$. Subsequently, cells were stained with FITC-Annexin V/PI using a cell apoptosis detection kit (BD Biosciences) for $30 \mathrm{~min}$ in the dark according to the manufacturer's protocol. Subsequently, $330 \mu \mathrm{l}$ binding buffer was added to each sample and apoptosis of cells was analyzed using a flow cytometer (CytoFlex; Beckman Coulter, Inc.). The apoptotic cells were defined as Annexin V-positive and PI-negative and data were analyzed using Kaluza Analysis Software v2 (Beckman Coulter, Inc.).

Wound healing assay. A549 cells or NCI-1650 cells were seeded into 6-well plates and cultured until they reached $100 \%$ confluency. The monolayer of cells was scratched using a 200- $\mu 1$ sterile pipette tip, and the culture was continued for another $48 \mathrm{~h}$ in serum-free medium. Wound closure was imaged at 0 and $48 \mathrm{~h}$ using a light microscope (Nicon Eclipase E600; Nikon Corporation). The width of the wound was measured using ImageJ (version $1.48 \mathrm{u}$; National Institutes of Health), and the relative wound width was calculated to indicate cell migration.
Transwell assay. A Transwell assay was performed to evaluate the migration and invasion of A549 cells or NCI-1650 cells using a Transwell chamber with or without pre-coated Matrigel (8- $\mu \mathrm{m}$ pore size; Corning, Inc.). For pre-coating, Matrigel was moved from $-20^{\circ} \mathrm{C}$ to $4^{\circ} \mathrm{C}$ overnight and diluted with serum-free DMEM (1:5) on ice with a pre-cooled pipette. Subsequently, an equal volume of diluted Matrigel was added to the upper transwell chamber for coverage. After incubation at $37^{\circ} \mathrm{C}$ for $1 \mathrm{~h}$, the coated chamber was rinsed with serum-free DMEM to remove the uncombined Matrigel, followed by addition of $50 \mu \mathrm{l}$ serum-free medium with $10 \mathrm{~g} / 1 \mathrm{BSA}$ at $37^{\circ} \mathrm{C}$. A total of $5 \times 10^{4}$ cells were suspended in serum-free DMEM and seeded into the upper chamber. Subsequently, $500 \mu 1$ DMEM supplemented with $10 \%$ FBS was added to the lower chamber. After incubation at $37^{\circ} \mathrm{C}$ for $48 \mathrm{~h}$, the migrated or invaded cells were fixed with $4 \%$ formaldehyde at room temperature for $20 \mathrm{~min}$, stained with $1 \%$ crystal violet at room temperature for $15 \mathrm{~min}$, and counted under a light microscope (Nicon Eclipase E600; Nikon Corporation).

Xenograft tumor model. A total of $10 \mathrm{BALB} / \mathrm{c}$ male nude mice (age, 6 weeks; weight, 18-20 g), were purchased from Shanghai SLAC Laboratory Animal Co., Ltd. and housed in a specific pathogen-free room with a controlled humidity of $40-60 \%$, a temperature of $24-26^{\circ} \mathrm{C}$, a $12 / 12 \mathrm{~h} \mathrm{light/dark} \mathrm{cycle,} \mathrm{and} \mathrm{free}$ access to food and water. After adaptation for 1 week, the xenograft tumor model was established by subcutaneously inoculating A549 cells $\left(5 \times 10^{6}\right)$ stably transfected with sh-MUC13 or scrambled shRNA in PBS into the right flank of the mice (age, 7 weeks). Tumor growth was measured every 5 days. On day 25 , the mice were euthanatized with $\mathrm{CO}_{2}$ exposure at a flow rate of $30 \%$ volume per minute, and death was confirmed by continued $\mathrm{CO}_{2}$ exposure for at least $15 \mathrm{~min}$ after breathing stopped. The xenograft tumors were dissected, weighed, and fixed with $4 \%$ formaldehyde at $4^{\circ} \mathrm{C}$ for $>24$ h or stored at $-80^{\circ} \mathrm{C}$. All animal experiments were performed according to the guidelines of the Chinese Experimental Animal Administration Legislation and approved by the Institutional Animal Care and Use Committee of Gansu Provincial Hospital (Lanzhou, China; approval no. RMYYLAC202033-2).

Immunohistochemistry (IHC) staining. Xenograft tumor tissues or patient tissue samples were washed twice with PBS and cut into pieces of the appropriate size $\left(5 \mathrm{~mm}^{3}\right)$. Subsequently, the tissues were fixed with $4 \%$ formaldehyde at $4{ }^{\circ} \mathrm{C}$ for 2 days and dehydrated using gradient ethanol. Next, the tissues were paraffin-embedded at $60^{\circ} \mathrm{C}$ overnight and cut into $5-\mu \mathrm{m}$ thick sections. The sections were then heated at $60^{\circ} \mathrm{C}$ for $1 \mathrm{~h}$, de-waxed using xylene, and rehydrated with gradient alcohol solution (100, 95,80 and $70 \%$ for 2 min each at room temperature) followed by antigen retrieval in a microwave oven in boiled $0.01 \mathrm{M}$ sodium citrate buffer ( $\mathrm{pH}$ 6.0) three times for 3 min each with $5 \mathrm{~min}$ intervals. This was followed by the addition of $3 \% \mathrm{H}_{2} \mathrm{O}_{2}$ to the sections for $10 \mathrm{~min}$ to block endogenous peroxidase, after which the sections were washed three times with PBS. Next, the sections were blocked with $5 \%$ goat serum (Beyotime Institute of Biotechnology) at room temperature for $1 \mathrm{~h}$, followed by incubation overnight at $4^{\circ} \mathrm{C}$ with anti-Ki-67 antibodies (dilution, 1:200; cat. no. ab15580; Abcam). After washing with PBS three times, sections were then incubated with donkey anti-rabbit 


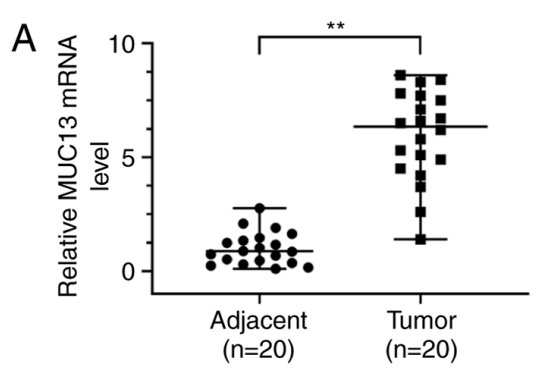

C

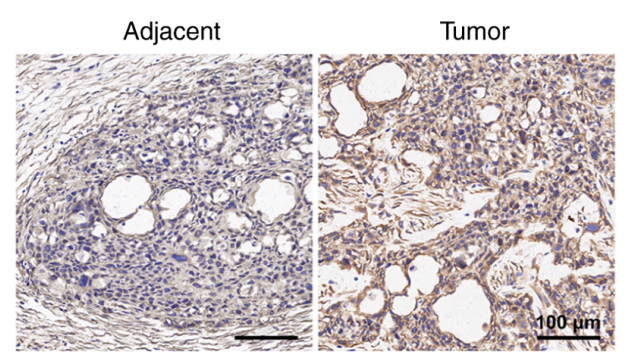

D MUC13 with 526 cancer and 59 normal samples in LUAD

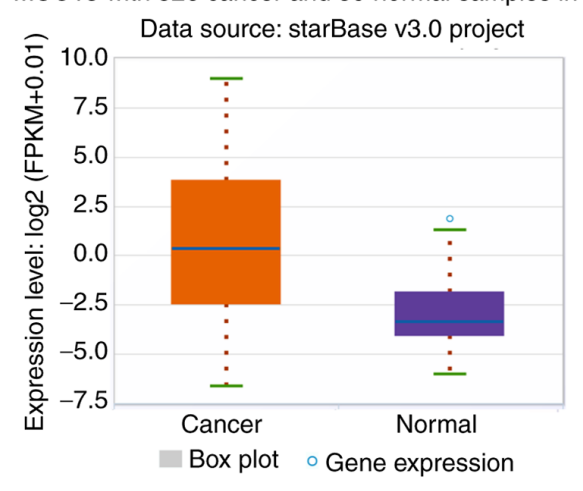

B

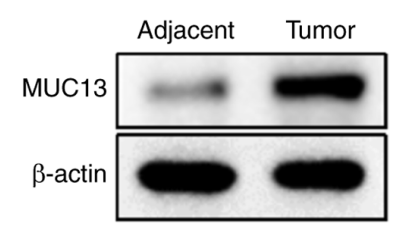

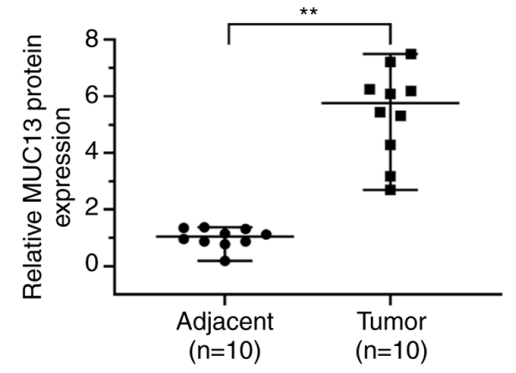

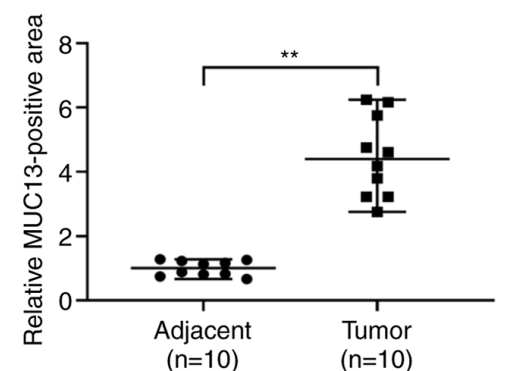

$\mathrm{E}$

Figure 1. MUC13 expression is increased in lung cancer. (A) mRNA expression levels of MUC13 in lung tumor tissues and adjacent normal tissues were analyzed by RT-qPCR. (B) Paired lung cancer and normal control tissues were analyzed by western blotting and a representative example is presented in this figure. (C) Immunohistochemical staining was performed to examine MUC13 expression in lung tumor and adjacent normal control tissues. Scale bar, $100 \mu \mathrm{m}$. (D) Expression levels of MUC13 in 526 cancer and 59 normal samples in lung adenocarcinoma were analyzed using StarBase datasets. (E) mRNA expression levels of MUC13 in lung cancer cell lines (NCI-H460, H1703, A549 and NCI-H1650), control cells (NLECs) and HBEs were analyzed by RT-qPCR (n=3). ${ }^{* *} \mathrm{P}<0.01$. FPKM, fragments per kilo base per million mapped reads; HBEs, human bronchial epithelial cells; LUAD, lung adenocarcinoma; MUC13, mucin 13; NLECs, normal lung epithelial cells; RT-qPCR, reverse transcription-quantitative PCR.

secondary antibody (1:500; cat. no. ab207999; Abcam) the next day at room temperature for $45 \mathrm{~min}$. Biotin-labeled HRP was added to the tissue sections and the staining was visualized using a DAB Substrate Kit (cat. no. ab64238; Abcam). Following this, sections were counterstained with hematoxylin for nuclei staining at room temperature for 5-10 $\mathrm{min}$. Then, slices were washed with running water, dehydrated with ethanol, hyalinized by xylene, mounted with neutral resins (cat. no. E675007; Sangon Biotech Co., Ltd.) and analyzed under a light microscope (Nicon Eclipase E600; Nikon Corporation). To evaluate MUC13 as a transmembrane glycoprotein, the MUC13-positive area was calculated for three different fields and its expression was recorded as a percentage of the total area of the field using ImageJ software (version 1.48u; National Institutes of Health). To detect Ki-67 in the nucleus, the number of Ki-67-positive cells was calculated for three different fields and the number of positive cells per field was recorded for analysis.

Statistical analysis. Results are presented as the mean \pm SD of at least three experiments. The statistical analysis was conducted using GraphPad Prism (V6; GraphPad Software, Inc.). Student's paired t-test was used for comparisons between clinical samples and unpaired Student's t-test was performed for comparisons between two groups of cell samples. One-way ANOVA followed by Tukey's post hoc test was used for comparisons among multiple groups. $\mathrm{P}<0.05$ was considered to indicate a statistically significant difference.

\section{Results}

MUC13 is highly expressed in lung cancer. Previous studies have demonstrated that MUC13 is abnormally expressed in multiple malignancies (9-12). In the present study, the expression levels of MUC13 were first analyzed in lung tumor tissues and adjacent normal tissues. RT-qPCR demonstrated that the expression levels of MUC13 in lung tumor tissues were significantly higher than those in the adjacent normal tissues (Fig. 1A). Western blotting also demonstrated that MUC13 protein expression was increased in lung tumor tissues compared with that in adjacent normal tissues (Fig. 1B). 
A $\begin{array}{ll}\text { Scramble } & \text { MUC13 shRNA-2 } \\ \text { MUC13 shRNA-1 } & \text { MUC13 shRNA-3 }\end{array}$

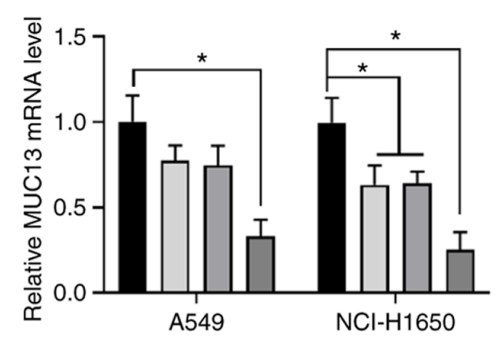

C

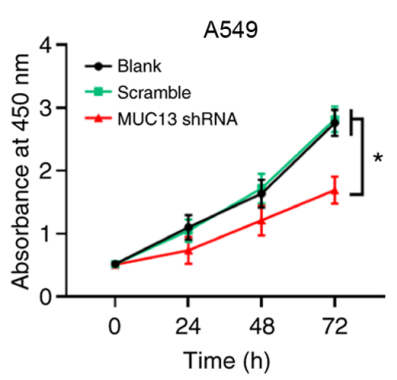

E

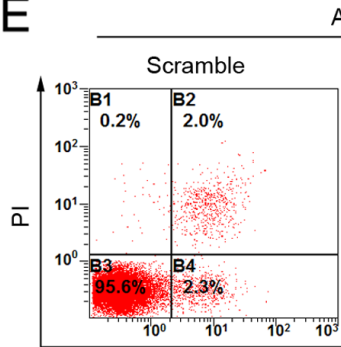

549

B

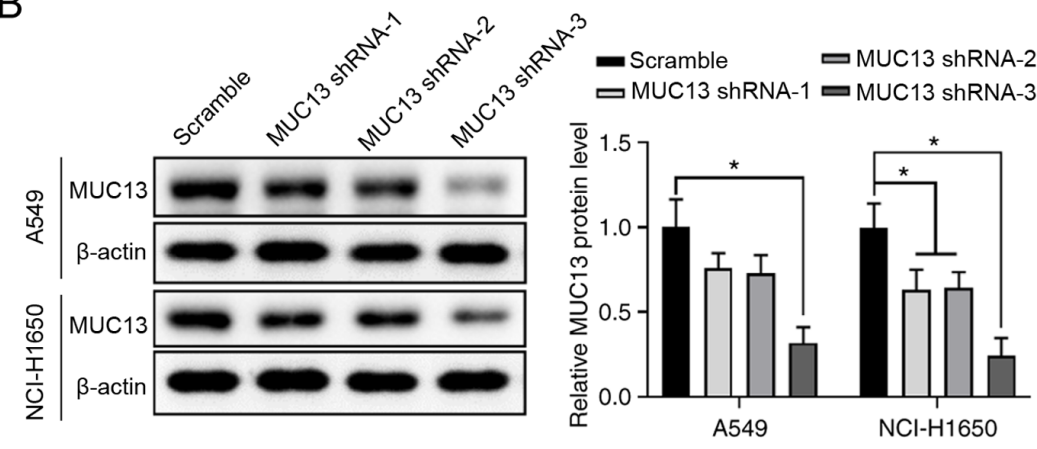

D
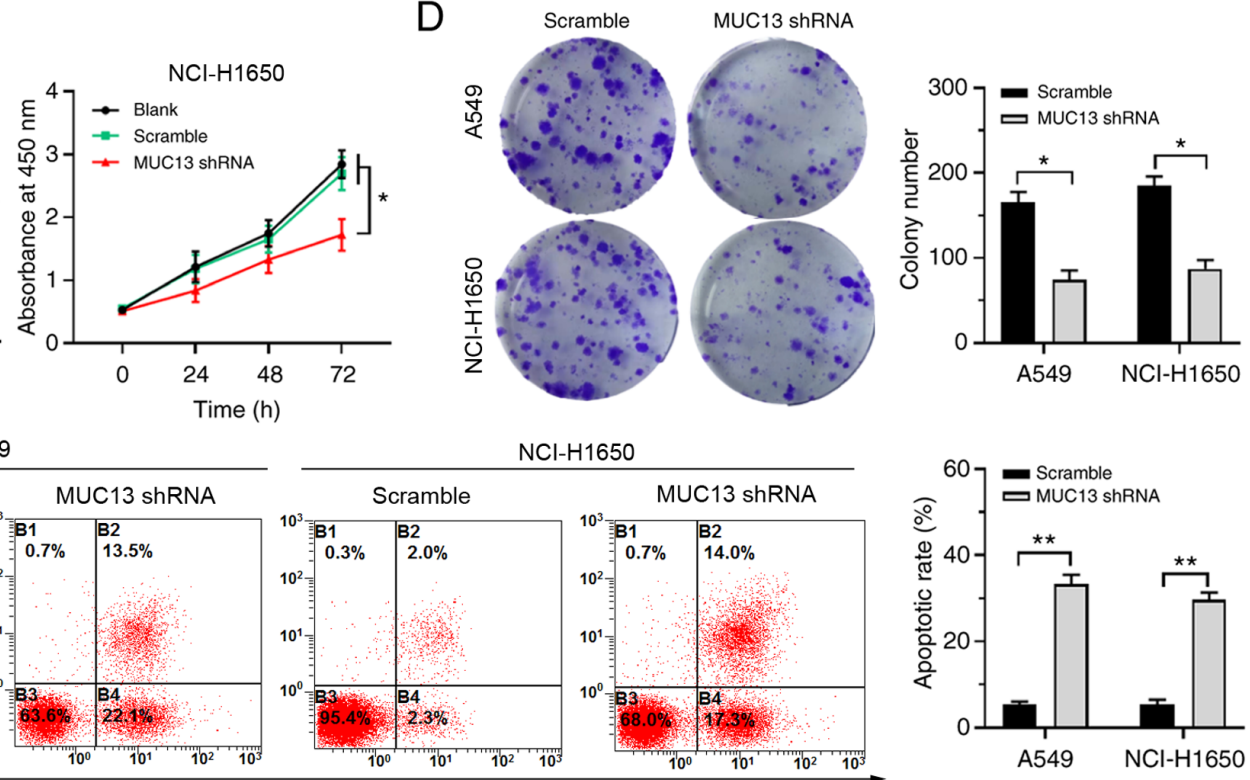

$\mathrm{NCl}-\mathrm{H} 1650$

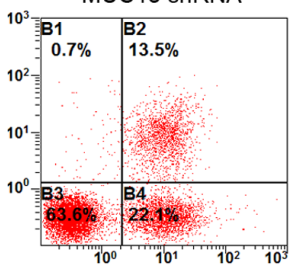

Annexin V-FITC

Figure 2. Knockdown of MUC13 inhibits cell proliferation and promotes the apoptosis of lung cancer cells. (A and B) A549 or NCI-H1650 cells were transfected with scramble control or shRNA targeting MUC13. (A) mRNA and (B) protein expression levels of MUC13 were analyzed $48 \mathrm{~h}$ later by reverse transcription-quantitative PCR and western blotting. (C-E) A549 or NCI-H1650 cells were transfected with scramble control or MUC13 shRNA-3. (C) Cell proliferation was analyzed using a Cell Counting Kit-8 assay. (D) Colony numbers were evaluated using a colony formation assay. (E) Cell apoptosis was analyzed using Annexin V/PI double staining. $n=3 .{ }^{*} \mathrm{P}<0.05,{ }^{* *} \mathrm{P}<0.01$. MUC13, mucin 13; shRNA, short hairpin RNA.

Furthermore, IHC analysis revealed that MUC13 expression was upregulated in lung tumor tissues compared with that in adjacent normal tissues (Fig. 1C). MUC13 expression was also analyzed and compared between 526 cancer samples and 59 normal samples derived from patients with lung adenocarcinoma using StarBase datasets. Consistently, the results demonstrated that MUC13 expression was significantly upregulated in lung cancer samples (Fig. 1D). Furthermore, MUC13 expression was markedly higher in lung cancer cell lines than in control NLECs; however, but no significant difference was identified between NLECs and HBE cells (Fig. 1E). These findings suggested that the expression levels of MUC13 were abnormally upregulated in lung cancer.

Knockdown of MUC13 inhibits proliferation and promotes apoptosis of lung cancer cells. To investigate the function of MUC13 in lung cancer development, MUC13 expression was silenced in A549 or NCI-H1650 cells using the shRNA method. Both RT-qPCR and western blotting demonstrated that MUC13 shRNA-1 and shRNA-2 could only slightly suppress MUC13 expression in NCI-1650 cells but exerted no obvious influence on MUC13 expression in A549 cells, whereas MUC13 shRNA-3 could significantly suppress MUC13 expression compared with that in the scramble group in both A549 and NCI-H1650 cells (Fig. 2A and B). Therefore, the most efficient shRNA (MUC13 shRNA-3) was used for subsequent experiments. The results of CCK-8 and colony formation assays demonstrated that knockdown of MUC13 inhibited the proliferation and colony formation of A549 and NCI-H1650 cells (Fig. 2C and D). Furthermore, Annexin V/PI double-staining revealed that silencing of MUC13 expression could significantly increase the apoptosis of A549 and NCI-H1650 cells (Fig. 2E). These results demonstrated that inhibiting MUC13 expression could significantly suppress the proliferation and promote the apoptosis of lung cancer cells.

Knockdown of MUC13 suppresses the migration and invasion of lung cancer cells. The effect of MUC13 on the migration and invasion of lung cancer cells was also determined. The results of a wound-healing assay revealed that knockdown of MUC13 significantly suppressed the wound width reduction of A549 and NCI-H1650 cells compared with the scramble group at $48 \mathrm{~h}$ (Fig. 3A). Consistently, the Transwell assays also revealed that knockdown of MUC13 decreased the 

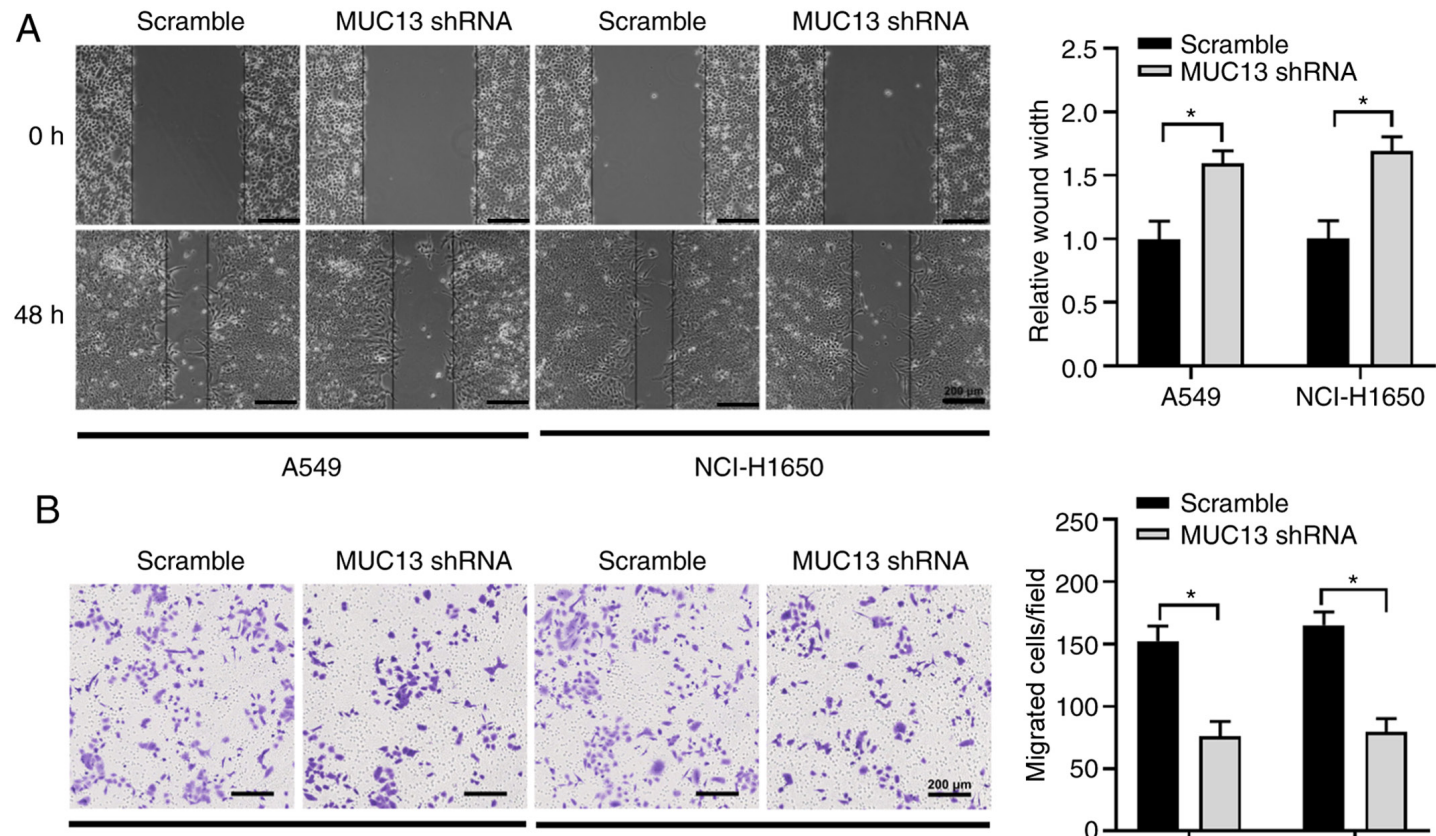

A549
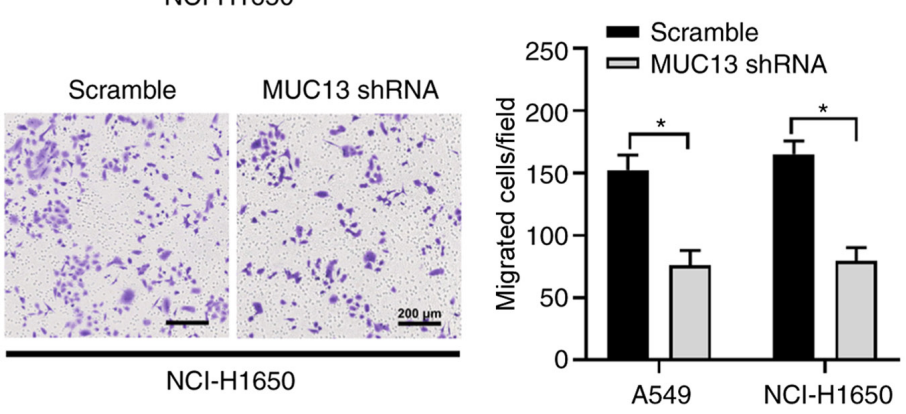

C

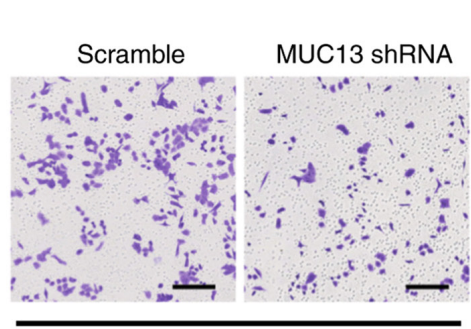

A549

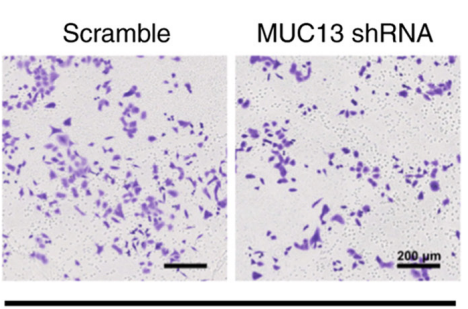

$\mathrm{NCl}-\mathrm{H} 1650$

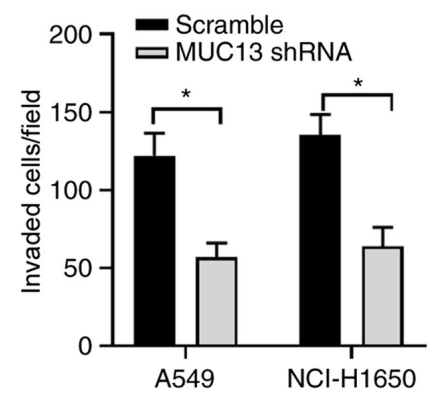

Figure 3. Knockdown of MUC13 suppresses the migration and invasion of lung cancer cells. A549 or NCI-H1650 cells were transfected with scramble control or MUC13 shRNA. (A) Wound healing assays were performed to evaluate cell migration. Scale bar, $200 \mu \mathrm{m}$. Transwell assays were performed to evaluate cell (B) migration and (C) invasion. Scale bar, $200 \mu \mathrm{m} . \mathrm{n}=3$. "P<0.05. MUC13, mucin 13; shRNA, short hairpin RNA.

migration and invasion of lung cancer cells (Fig. 3B and C). This suggested that silencing MUC13 could markedly inhibit the migration and invasion of lung cancer cells.

Knockdown of MUC13 inhibits xenograft lung tumor development in vivo. To further explore the function of MUC13 in lung cancer development, a xenograft tumor model was established by subcutaneous injection of A549 cells with stable knockdown of MUC13 or injection of control cells. Silencing of MUC13 expression significantly delayed the increase of tumor volume in vivo compared with the scramble group (Fig. 4A). The weights of tumors collected from the MUC13-knockdown group were lower than those from the control group (Fig. 4B). Furthermore, IHC staining of the proliferation marker Ki-67 demonstrated that knockdown of MUC13 inhibited the proliferation of lung cancer cells in vivo, as indicated by lower Ki-67 expression (Fig. 4C). Overall, these results indicated that knockdown of MUC13 could markedly inhibit the growth of xenograft lung tumors.

MUC13 promotes lung cancer development via regulation of ERK signaling. To further examine the mechanism of MUC13 regulation in lung cancer, ERK, an underlying signaling pathway of MUC13 (14), was evaluated in the present study.
Compared with those in the adjacent control tissues, the phosphorylation levels of ERK, JNK and p38 in the lung cancer tissues were significantly increased (Fig. 5A). By contrast, knockdown of MUC13 in A549 and NCI-H1650 cells markedly decreased the levels of p-ERK, p-JNK and p-p38 (Fig. 5B). Overall, these findings suggested that MUC13 might promote lung cancer development via regulation of ERK signaling.

\section{Discussion}

The oncogenic role of MUC13 in regulating cancer cell proliferation, apoptosis, migration and invasion has been reported in various malignancies, including pancreatic cancer and intraductal papillary mucinous neoplasms $(21,22)$. However, its role in lung cancer remains unknown. The results of the present study demonstrated that MUC13 expression was upregulated in lung cancer cells and that knockdown of MUC13 suppressed lung cancer cell proliferation, migration and invasion, which may further help elucidate the mechanism of lung cancer development.

Upregulation of MUC13 expression has been identified in pancreatic cancer, and MUC13 could enhance cell motility and proliferation via activation of p21-activated kinase 1 and ERK signaling (11). In colon cancer cells, upregulated MUC13 

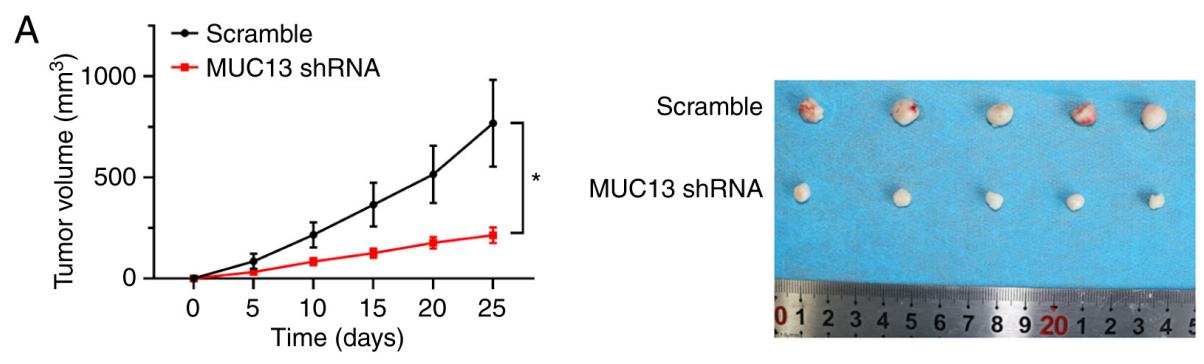

C

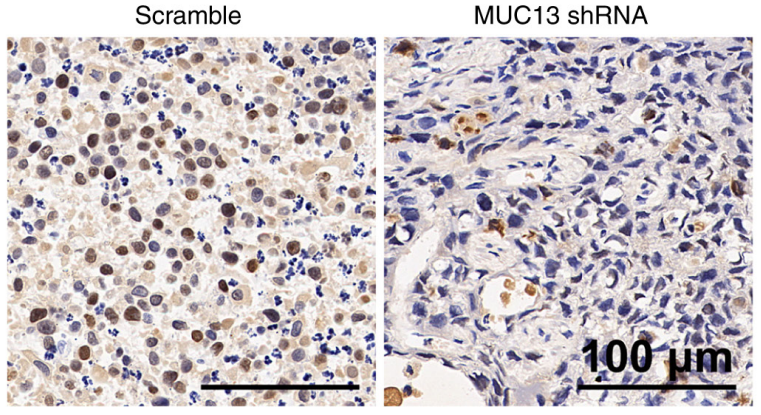

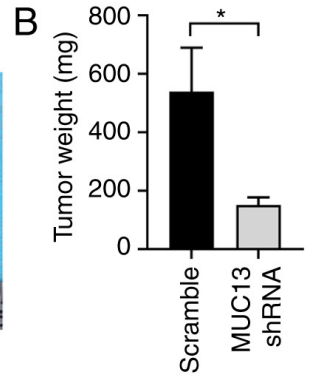

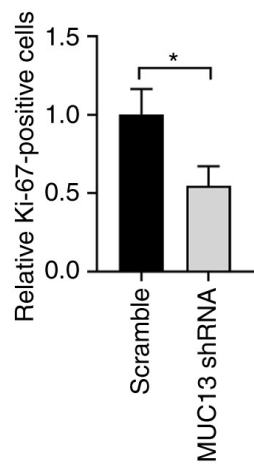

Figure 4. Knockdown of MUC13 inhibits xenograft lung tumor development in vivo. A549 cells were stably transfected with MUC13 shRNA or scramble control and then subcutaneously injected into nude mice. (A) Tumor growth was monitored and xenograft tumors from different groups were imaged. (B) Tumor weights were analyzed. (C) Immunohistochemical staining was performed to examine Ki-67 expression in tumor tissue sections. Scale bar, $100 \mu \mathrm{m}$. $\mathrm{n}=5$. ${ }^{*} \mathrm{P}<0.05$. MUC13, mucin 13; shRNA, short hairpin RNA.
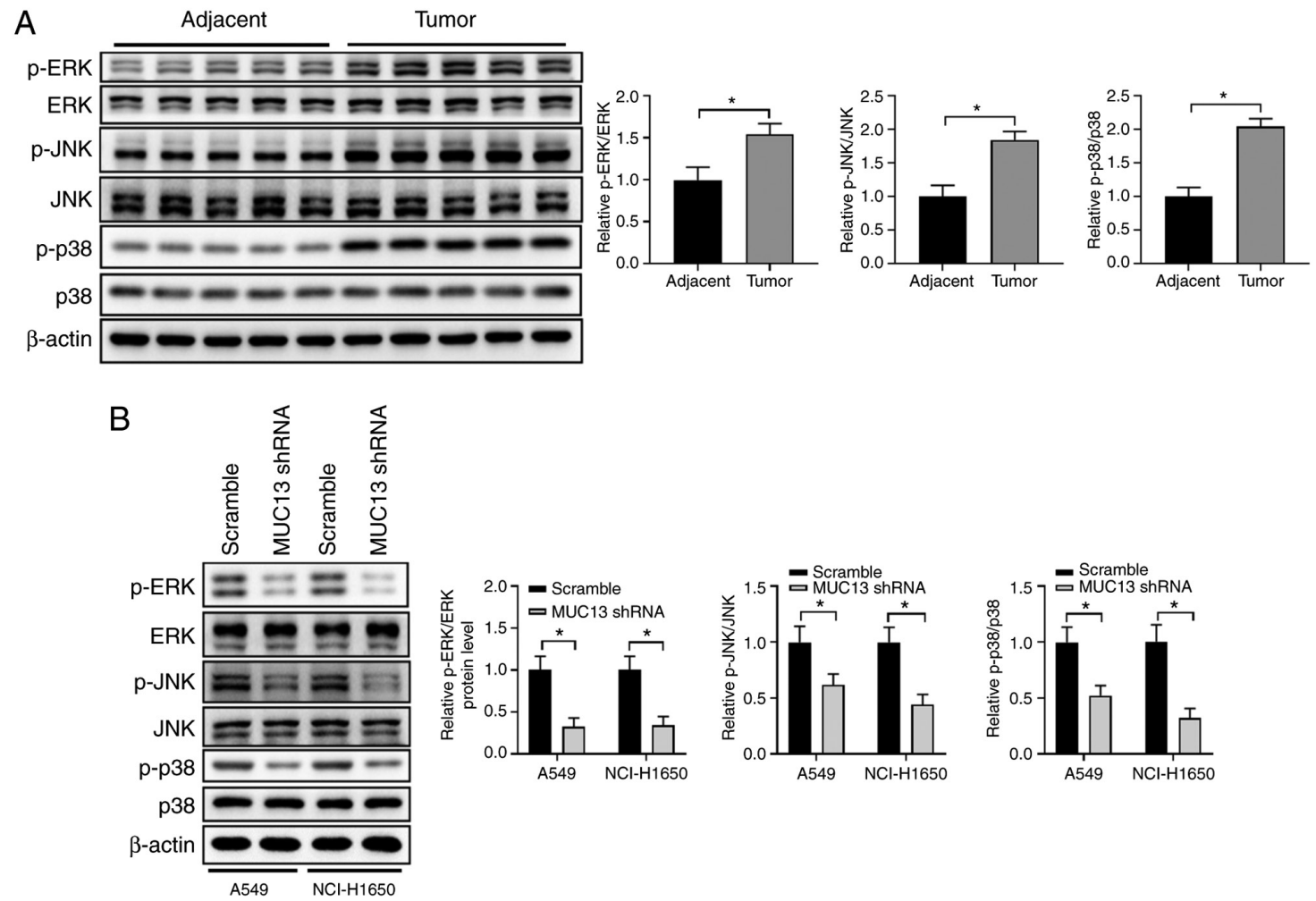

Figure 5. MUC13 promotes lung cancer development via regulation of ERK signaling. (A) Western blotting was performed to analyze the levels of p-ERK/ERK, p-JNK/JNK and p-p38/p38 in lung tumor tissues and adjacent normal tissues. (B) A549 or NCI-H1650 cells were transfected with scramble control or MUC13 shRNA. The levels of p-ERK/ERK, p-JNK/JNK and p-p38/p38 were analyzed by western blotting. $\mathrm{n}=5$. * $\mathrm{P}<0.05$. MUC13, mucin 13 ; p-, phosphorylated; shRNA, short hairpin RNA.

expression has been reported to enhance the tumorigenic features of colon cancer cells and to increase the phosphorylation of HER2 and ERK (12). Similarly, the present study revealed that lung cancer cells exhibited enhanced phosphorylation of ERK, JNK and p38, whereas knockdown of MUC13 significantly suppressed the phosphorylation of ERK, JNK 
and p38 in lung cancer cells. Additionally, Sheng et al (23) demonstrated that MUC13 promotes renal cell carcinoma progression and drug resistance by inducing the cell cycle regulator cyclin D1, and that it inhibits cell apoptosis by inducing Bcl-XL and survivin expression. Whether MUC13 regulates the cell cycle in lung cancer cells requires further investigation.

Since MUC13 functions as an oncogenic glycoprotein in multiple malignancies, including hepatocellular carcinoma, colorectal cancer and esophageal squamous cell carcinoma (24-26), it is of significant interest to explore the regulation of MUC13 in tumors. A chromatin immunoprecipitation assay has demonstrated that the transcription factor upstream transcription factor 1 could bind to the promoter region of MUC13 and enhance MUC13 expression in glioblastoma (27). Additionally, the expression levels of MUC13 are regulated post-transcriptionally by miR-145 in pancreatic cancer and colorectal cancer (15). In another study, the inhibition of miR-132-3p led to elevated expression levels of MUC13 and enhanced gastric cancer cell proliferation (16). However, the mechanism by which MUC13 expression is regulated in lung cancer development requires further investigation. A previous study demonstrated that patients with high MUC13 expression in the cytoplasm and nucleus presented with a larger colon tumor size and poorly differentiated grade compared with the patients with only membrane localization (28). In addition, MUC13 is upregulated and co-localized with parasites during hepatic infection, and could be a hallmark of Plasmodium exoerythrocytic infection (29). Considering these aforementioned results (24-28), it is of significance to detect the subcellular location and downstream target of MUC13 in subsequent investigations to further clarify the mechanism of MUC13 in lung cancer.

In conclusion, MUC13 functions as an oncogenic glycoprotein mucin in the development of lung cancer via activation of downstream ERK/JNK/p38 signaling. The results of the present study suggested that MUC13 served a vital role in the progression of lung cancer. It is important to further elucidate the function and mechanism of MUC13 in lung cancer, and this may provide novel insights for improving the understanding and treatment of the disease.

\section{Acknowledgements}

Not applicable.

\section{Funding}

No funding was received.

\section{Availability of data and materials}

The datasets used and/or analyzed during the current study available from the corresponding author on reasonable request.

\section{Authors' contributions}

YP, YZ and ZJZ participated in the conception and design of the study. HYZ and WHW performed the research. GJ and JWL performed the statistical analyses and evaluated the results. YZ drafted the paper. YP and ZJZ contributed to the enrollment of patients and analysis of data. YP, YZ and ZJZ confirmed the authenticity of all raw data. All authors read and approved the final manuscript.

\section{Ethics approval and consent to participate}

Written informed consent was obtained from all patients and the study protocol was approved by the Ethics Committee of Gansu Provincial Hospital (Lanzhou, China). Animal care and experiments were approved by the Institutional Animal Care and Use Committee of Gansu Provincial Hospital.

\section{Patient consent for publication}

Not applicable.

\section{Competing interests}

The authors declare that they have no competing interests.

\section{References}

1. Bade BC and Dela Cruz CS: Lung Cancer 2020: Epidemiology, Etiology, and Prevention. Clin Chest Med 41: 1-24, 2020.

2. Yang D, Liu Y, Bai C, Wang X and Powell CA: Epidemiology of lung cancer and lung cancer screening programs in China and the United States. Cancer Lett 468: 82-87, 2020.

3. Hirsch FR, Scagliotti GV, Mulshine JL, Kwon R, Curran WJ Jr, Wu YL and Paz-Ares L: Lung cancer: Current therapies and new targeted treatments. Lancet 389: 299-311, 2017.

4. Liu WJ, Du Y, Wen R, Yang M and Xu J: Drug resistance to targeted therapeutic strategies in non-small cell lung cancer. Pharmacol Ther 206: 107438, 2020.

5. Yan X,Jiao SC,Zhang GQ, Guan Y and Wang JL:Tumor-associated immune factors are associated with recurrence and metastasis in non-small cell lung cancer. Cancer Gene Ther 24: 57-63, 2017.

6. Hung JJ, Jeng WJ, Hsu WH, Chou TY, Huang BS and Wu YC: Predictors of death, local recurrence, and distant metastasis in completely resected pathological stage-I non-small-cell lung cancer. J Thorac Oncol 7: 1115-1123, 2012.

7. Williams SJ, Wreschner DH, Tran M, Eyre HJ, Sutherland GR and McGuckin MA: Muc13, a novel human cell surface mucin expressed by epithelial and hemopoietic cells. J Biol Chem 276: 18327-18336, 2001.

8. Sheng YH, Lourie R, Lindén SK, Jeffery PL, Roche D, Tran TV, Png CW, Waterhouse N, Sutton P, Florin TH, et al: The MUC13 cell-surface mucin protects against intestinal inflammation by inhibiting epithelial cell apoptosis. Gut 60: 1661-1670, 2011.

9. Shimamura T, Ito H, Shibahara J, Watanabe A, Hippo Y, Taniguchi H, Chen Y, Kashima T, Ohtomo T, Tanioka F, et al: Overexpression of MUC13 is associated with intestinal-type gastric cancer. Cancer Sci 96: 265-273, 2005.

10. Chauhan SC, Vannatta K, Ebeling MC, Vinayek N, Watanabe A, Pandey KK, Bell MC, Koch MD, Aburatani H, Lio Y, et al: Expression and functions of transmembrane mucin MUC13 in ovarian cancer. Cancer Res 69: 765-774, 2009.

11. Chauhan SC, Ebeling MC, Maher DM, Koch MD, Watanabe A, Aburatani H, Lio Y and Jaggi M: MUC13 mucin augments pancreatic tumorigenesis. Mol Cancer Ther 11: 24-33, 2012.

12. Gupta BK, Maher DM, Ebeling MC, Stephenson PD, Puumala SE, Koch MR, Aburatani H, Jaggi M and Chauhan SC: Functions and regulation of MUC13 mucin in colon cancer cells. J Gastroenterol 49: 1378-1391, 2014.

13. Sheng YH, He Y, Hasnain SZ, Wang R, Tong H, Clarke DT, Lourie R, Oancea I, Wong KY, Lumley JW, et al: MUC13 protects colorectal cancer cells from death by activating the NF- $\kappa B$ pathway and is a potential therapeutic target. Oncogene 36: 700-713, 2017.

14. Tiemin P, Fanzheng M, Peng X, Jihua H, Ruipeng S, Yaliang L, Yan W, Junlin X, Qingfu L, Zhefeng H, et al: MUC13 promotes intrahepatic cholangiocarcinoma progression via EGFR/PI3K/AKT pathways. J Hepatol 72: 761-773, 2020. 
15. Khan S, Ebeling MC, Zaman MS, Sikander M, Yallapu MM, Chauhan N, Yacoubian AM, Behrman SW, Zafar N, Kumar D, et al: MicroRNA-145 targets MUC13 and suppresses growth and invasion of pancreatic cancer. Oncotarget 5: 7599-7609, 2014.

16. He L, Qu L, Wei L, Chen Y and Suo J: Reduction of miR 132 3p contributes to gastric cancer proliferation by targeting MUC13. Mol Med Rep 15: 3055-3061, 2017.

17. Moniaux N, Escande F, Porchet N, Aubert JP and Batra SK: Structural organization and classification of the human mucin genes. Front Biosci 6: D1192-D1206, 2001.

18. Filippou PS, Ren AH, Korbakis D, Dimitrakopoulos L, Soosaipillai A, Barak V, Frenkel S, Pe'er J, Lotem M, Merims S, et al: Exploring the potential of mucin 13 (MUC13) as a biomarker for carcinomas and other diseases. Clin Chem Lab Med 56: 1945-1953, 2018.

19. Lim W, Ridge CA, Nicholson AG and Mirsadraee S: The 8th lung cancer TNM classification and clinical stageing system: Review of the changes and clinical implications. Quant Imaging Med Surg 8: 709-718, 2018

20. Livak KJ and Schmittgen TD: Analysis of relative gene expression data using real-time quantitative PCR and the 2(- $\Delta \Delta \mathrm{C}(\mathrm{T}))$ Method. Methods 25: 402-408, 2001.

21. Kumari S, Khan S, Gupta SC, Kashyap VK, Yallapu MM Chauhan SC and Jaggi M: MUC13 contributes to rewiring of glucose metabolism in pancreatic cancer. Oncogenesis 7: 19,2018

22. Stiles ZE, Khan S, Patton KT, Jaggi M, Behrman SW and Chauhan SC: Transmembrane mucin MUC13 distinguishes intraductal papillary mucinous neoplasms from non-mucinous cysts and is associated with high-risk lesions. HPB (Oxford) 21 87-95, 2019.

23. Sheng Y, Ng CP, Lourie R, Shah ET, He Y, Wong KY, Seim I, Oancea I, Morais C, Jeffery PL, et al: MUC13 overexpression in renal cell carcinoma plays a central role in tumor progression and drug resistance. Int J Cancer 140: 2351-2363, 2017.
24. Dai Y, Liu L, Zeng T, Liang JZ, Song Y, Chen K, Li Y, Chen L, Zhu YH, Li J, et al: Overexpression of MUC13, a poor prognostic predictor, promotes cell growth by activating Wnt signaling in hepatocellular carcinoma. Am J Pathol 188: 378-391, 2018.

25. Doxtater K, Sekhri R, Mishra U, Jaggi M, Tripathi M and Chauhan S: MUC13 enhances anchorage independent survival and cooperates with YAP1 and $\beta$-catenin towards colorectal cancer metastasis. Cancer Res 80 (Suppl): 4916, 2020.

26. Wang H, Shen L, Lin Y, Shi Q, Yang Y and Chen K: The expression and prognostic significance of Mucin 13 and Mucin 20 in esophageal squamous cell carcinoma. J Cancer Res Ther 11 (Suppl 1): C74-C79, 2015.

27. Li P, Wang H, Hou M, Li D and Bai H: Upstream stimulating factor1 (USF1) enhances the proliferation of glioblastoma stem cells mainly by activating the transcription of mucin13 (MUC13). Pharmazie 72: 98-102, 2017.

28. Gupta BK, Maher DM, Ebeling MC, Sundram V, Koch MD, Lynch DW, Bohlmeyer T, Watanabe A, Aburatani H, Puumala SE, et al: Increased expression and aberrant localization of mucin 13 in metastatic colon cancer. J Histochem Cytochem 60: 822-831, 2012

29. LaMonte GM, Orjuela-Sanchez P, Calla J, Wang LT, Li S, Swann J, Cowell AN, Zou BY, Abdel-Haleem Mohamed AM, Villa Galarce ZH, et al: Dual RNA-seq identifies human mucosal immunity protein Mucin-13 as a hallmark of Plasmodium exoerythrocytic infection. Nat Commun 10: 488, 2019.

This work is licensed under a Creative Commons Attribution-NonCommercial-NoDerivatives 4.0 International (CC BY-NC-ND 4.0) License. 